\title{
B-cell lymphoma in a tubular adenoma with high- grade dysplasia: a rare extramedullary manifesta- tion of high-grade diffuse large B-cell lymphoma
}

B-cell lymphomas represent about $90 \%$ of all non-Hodgkin lymphomas (NHLs). Almost all express pan B-cell antigens including CD19, CD20, and PAX5. Most patients with NHL present persistent painless peripheral lymphadenopathy. Staging tests do not include colonoscopy. Here we present a rare case of synchronous occurrence of a high-grade diffuse large B-cell lymphoma (DLBCL) initially presenting in a tubular adenoma of the large bowel and subsequently in the bone marrow.

A 73-year-old woman was referred to our hospital because of leukopenia (minimum $0.6 \times 10^{9} / \mathrm{L}$ ), anemia (hemoglobin $83 \mathrm{~g} / \mathrm{L}$ ), dyspnea, and B symptoms. Previously, 27 years earlier, the patient suffered from a pluriform differentiated mucinous breast carcinoma (pT3 pN0 M0) treated with radiation (46 Gy high dose) after mastectomy.

A computed tomography (CT) scan of the abdomen detected few enhanced paraaortal lymph nodes, and routine laboratory analysis showed elevated values for lactate dehydrogenase (maximum $2892 \mathrm{U} / \mathrm{L})$, C-reactive protein $(312 \mathrm{mg} / \mathrm{L})$, liver enzymes, ferritin ( $6334 \mathrm{ng} / \mathrm{mL})$, and CA $12-5(160 \mathrm{U} / \mathrm{mL})$. Colonoscopy revealed a $2-\mathrm{cm}$ tubular adenomatous sigmoid polyp, which was completely removed by endoscopic resection ( $\bullet$ Fig. 1 ). Histological examination and appropriate immunohistochemical staining showed high-grade intraepithelial neoplasia and focal infiltration of the stroma by highly proliferative lymphoid blasts positive for CD20 and PAX5. Bone marrow immunocytology displayed a monoclonal B-cell lymphocytosis. Subsequent bone marrow biopsy revealed an interstitial and diffuse infiltration by a DLBCL with positivity for CD20, bcl2, and PAX5, negativity for TdT, CD10, and CD34, and a proliferation index of $80 \%$ (MIB1 staining). Infiltration by the previously diagnosed breast carcinoma was excluded by negativity for the pankeratin markers A/E1-3 and MNF116.

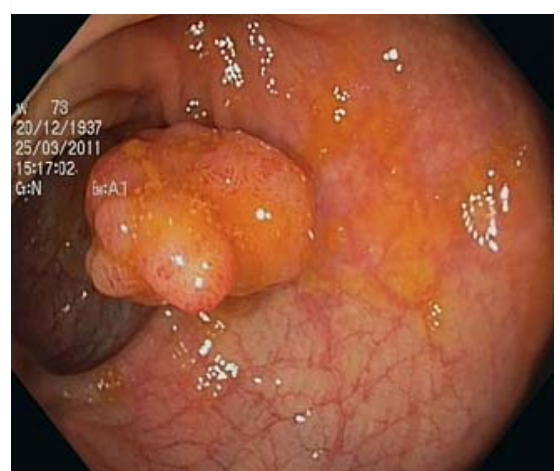

Fig. 1 Endoscopic view of the polyp at the rectosigmoid junction. Note the smooth contour of the mildly protruding lesion as well as the bleeding, which occurred even with gentle endoscopic manipulation.

Finally, molecular genetic analysis detecting the rearrangement of the FR3a region of the immunoglobulin heavy chain was performed, and identical monoclonal amplificates of approximately 248 base pairs were detected in both manifestations, demonstrating malignancy and clonal association of the lymphoma infiltrates in the adenoma and the bone marrow ( $\boldsymbol{O}$ Fig. 2 ). The ileocecal area and ileum are the regions most frequently affected by primary small-intestinal and large-intestinal NHL, and most of such cases are DLBCL [1-3]. Synchronous diagnosis of colorectal malignancy and lymphoma is rare [4]. Colorectal lymphoma is extremely infrequent, representing less than $0.5 \%$ of all primary colorectal neoplasms [5].

B-cell lymphomas should be included in the differential diagnosis of polypoid lesions in patients suspicious for malignancies. Endoscopy with complete histological analysis may be important in the diagnosis of high-grade DLBCL of the lower gastrointestinal tract.

\section{Endoscopy_UCTN_Code_CCL_1AD_2AC}

Competing interests: None
E. Roeb ${ }^{1}$, M. Rummel ${ }^{2}$, W. Blau ${ }^{2}$, B. Etschmann ${ }^{3}$, S. Gattenlöhner ${ }^{3}$ ${ }^{1}$ Division of Gastroenterology, Department of Internal Medicine, University Hospital, Justus Liebig University, Giessen, Germany

2 Division of Hematology, Department of Internal Medicine, Justus Liebig University, Giessen, Germany 3 Institute of Pathology, University Hospital, Justus Liebig University, Giessen, Germany

\section{References}

1 Bollen P, Bourgain C, Van Berlaer G et al. NonHodgkin lymphoma presenting as a solitary rectal polyp. J Pediatr Gastroenterol Nutr 2000; 31: 193- 194

2 Nakamura H, Fu KI, Matsumoto J et al. Polypectomy as a diagnostic tool for ileocecal lymphom. Endoscopy 2007; 39: 43

3 Kohno S, Ohshima K, Yoneda S et al. Clinicopathological analysis of 143 primary malignant lymphomas in the small and large intestines based on the new WHO classification. Histopathology 2003; 43: 135-143

4 Kidd L, Evans M, Williams N, Beynon J. Synchronous diagnosis of colorectal malignancy and lymphoma. Colorectal Dis 2010; [Epub ahead of print]

5 Marín García D, Cárdenas Lafuente F, Utrilla Ayala Mdel C et al. Primary diffuse large Bcell lymphoma of the rectum simulating a rectal adenocarcinoma. Gastroenterol Hepatol 2010; 33: $92-98$

\section{Bibliography}

Dol $10.1055 / \mathrm{s}-0030-1256841$

Endoscopy 2011; 43: E344 -E345

(c) Georg Thieme Verlag KG Stuttgart · New York . ISSN 0013-726X

\section{Corresponding author}

\section{E. Roeb, MD}

Division of Gastroenterology

Department of Internal Medicine II

Justus Liebig University Giessen

Clinic Street 33

D-35385 Giessen

Germany

Fax: +49-641-98542339

Elke.Roeb@innere.med.uni-giessen.de 


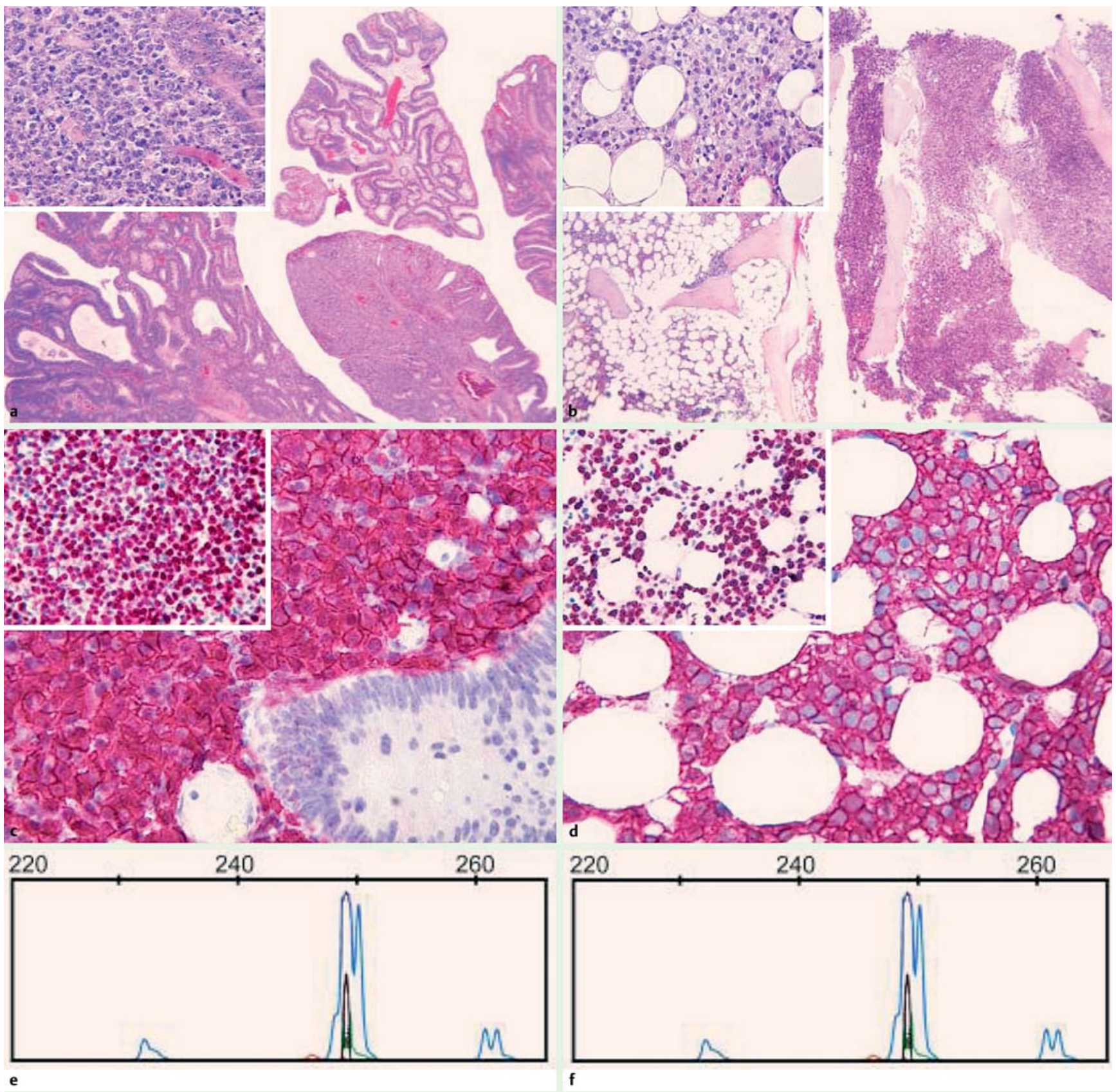

Fig. 2 Histochemical and molecular genetic analysis of lymphoma infiltrates in: a, c, e the colonic adenoma; b, d, $\mathbf{f}$ bone marrow biopsy. Lymphoid blast infiltrates in the adenomatous stroma (a, circle and inset) and the bone marrow (b) are strongly positive for the B-cell marker CD20 (c and d) and show proliferation rates $>80 \%$ with the MIB1 stain (insets $\mathbf{c}$ and $\mathbf{d}$ ). Molecular genetic analysis detecting the rearrangement of the FR3a region of the immunoglobulin heavy chain $(\mathrm{lgH})$ demonstrates monoclonal amplificates for both lymphoma infiltrates, showing monoclonal peaks (e and $\mathbf{f}$ ) with a so-called slight polyclonal background in the colonic adenoma (e), representing the reactive non-neoplastic B-cells in the surrounding tissue. 